\title{
Vitamin D and disease
}

A recent study demonstrates the power of combining chromatin immunoprecipitation followed by sequencing (ChIP-seq) with genome-wide association (GWA) study data sets to explore the molecular basis of complex disease.

Ramagopalan and colleagues used ChIP-seq to produce high-resolution maps of the genomic binding of the vitamin $\mathrm{D}$ receptor (VDR) - a ligand-activated transcription factor - in human lymphoblastoid cell lines, with and without active ligand. In the presence of ligand, they identified 2,776 binding sites, many of which are in regions associated with active chromatin, consistent with the expected role of VDR at gene regulatory elements.Vitamin $D$ has been linked to several diseases, particularly autoimmune diseases, but the basis for the link is unclear. The authors compared their ChIP-seq data with GWA study data sets for 47 common traits and found significant enrichment of VDR sites in associated genomic intervals for multiple sclerosis and type 1 diabetes, along with other autoimmune diseases, cancers and traits such as height. The results implicated VDR in the control of many genes that were not previously thought to be regulated by this transcription factor. In most cases, the disease-associated SNPs do not disrupt VDR-binding motifs, so further resequencing might be informative for pinpointing which genetic variants alter gene regulation. Interestingly, VDR binding is also enriched in regions with signatures of positive selection, so future studies might be aimed at investigating the role of vitamin $\mathrm{D}$ during human evolution.

Mary Muers

\section{ORIGINAL RESEARCH PAPER}

Ramagopalan, S. V. et al. A ChIP-seq defined genome-wide map of vitamin $\mathrm{D}$ receptor binding: associations with disease and evolution. Genome Res. 24 Aug 2010 (doi:10.1101/ gr.107920.110)

FURTHER READING Hawkins, R. D., Hon, G. C. \& Ren, B. Next-generation genomics: an integrative approach. Nature Rev. Genet. 11, 476-486 (2010)

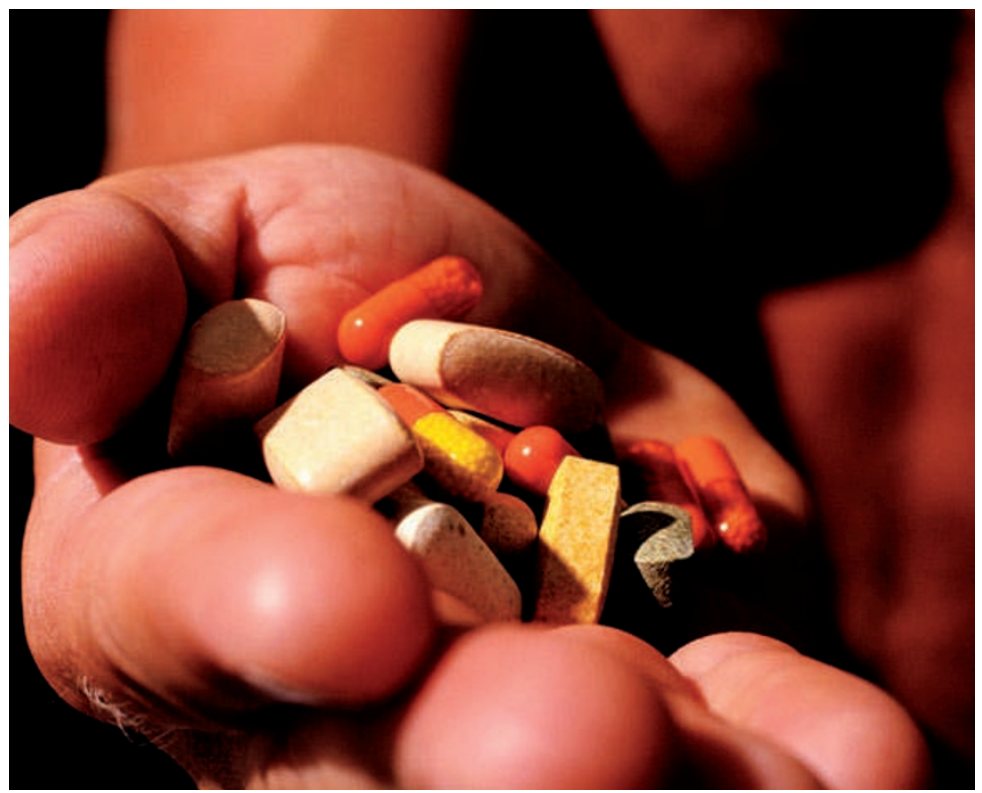

